\title{
石墨型 $\mathbf{C}_{3} \mathbf{N}_{4}$ 在泡沫陶瓷表面的原位负载及可见光催化 空气净化应用
}

\author{
崔雯 ${ }^{1}$ ，李茴 ${ }^{(1)}$ ，孙艳娟 ${ }^{(1)}$, 董帆 ${ }^{\left(1^{*}\right.}$, 何詠基 ${ }^{(2)}$ ，吴忠标 ${ }^{(3)}$ \\ (1) 重庆工商大学环境与资源学院, 催化与功能有机分子重庆市重点实验室, 重庆 400067; \\ (2) 香港教育学院科学与环境研究学系, 香港; \\ (3) 浙江大学环境工程系, 环境污染修复与生态健康教育部重点实验室, 杭州 310027 \\ * 联系人, E-mail: dfctbu@126.com
}

2015-08-26 收稿, 2015-09-22 接受, 2015-10-14 网络版发表

国家自然科学基金(51478070, 21501016, 51108487)和国家大学生创新创业训练计划(201411799004)资助

\begin{abstract}
摘要 $\mathrm{g}-\mathrm{C}_{3} \mathrm{~N}_{4}$ 是一种具有广泛应用前景的无金属可见光催化剂. 为了实现 $g-\mathrm{C}_{3} \mathrm{~N}_{4}$ 在载体表面的 有效负载, 本研究以二氯二胺为前驱体, 通过溶解-再结晶-热处理原位负载工艺, 利用 $\mathrm{g}_{-} \mathrm{C}_{3} \mathrm{~N}_{4}$ 和 $\mathrm{Al}_{2} \mathrm{O}_{3}$ 之间的独特物理化学作用力, 实现了 $\mathrm{Al}_{2} \mathrm{O}_{3}$ 泡沫陶瓷上 $\mathrm{g}-\mathrm{C}_{3} \mathrm{~N}_{4}$ 的牢固负载. 用 $\mathrm{X}$ 射线衍 射、扫描电子显微镜、透射电子显微镜、比表面积测定、紫外可见漫反射光谱和荧光光谱对样 品进行表征分析. 系统研究了热处理时间对样品微结构和光催化性能的影响. 结果表明, 当热 处理时间为 $4 \mathrm{~h}$ 时, 负载型 $\mathrm{g}-\mathrm{C}_{3} \mathrm{~N}_{4}$ 具有最优的 $\mathrm{NO}$ 去除率, 达 $57.9 \%$. 这可归因于其较大的比表面 积和较高的电荷分离效率. 本文提供了一种基于光催化剂与载体相互作用的原位负载技术, 为 g- $\mathrm{C}_{3} \mathrm{~N}_{4}$ 在空气净化的实际应用提供了技术支持和理论依据.
\end{abstract}

关键词

石墨型氮化碳

原位负载

可见光催化

电荷分离

空气净化
$\mathrm{NO}_{x}$ 是大气中重要污染物之一, 主要包括 $\mathrm{NO}$ 和 $\mathrm{NO}_{2}{ }^{[1]}$. $\mathrm{NO}_{x}$ 不仅是形成酸雨主要原因, 还会与非甲烷 碳氢化合物等发生光化学反应, 形成光化学烟雾 ${ }^{[2]}$. 此外, $\mathrm{NO}_{x}$ 会刺激呼吸器官, 引起急性和慢性中毒 ${ }^{[3]}$. 目前处理 $\mathrm{NO}_{x}$ 常用的方法有碱液吸收法 ${ }^{[4]}$ 、固体吸附 法 $^{[5]}$ 、选择性催化还原法 ${ }^{[6]}$ 、选择性非催化还原法 ${ }^{[7]}$ 、 生物净化法 ${ }^{[8]}$ 等, 然而这些技术仅适合高浓度 $\mathrm{NO}_{x}$ 的 处理, 对于环境空气中低浓度 $\mathrm{NO}_{x}(\mathrm{ppb}$ 级 $)$ 的处理效 率低, 且在经济上不可行 ${ }^{[9,10]}$.

可见光驱动的光催化是一种环境友好的绿色技 术, 在环境污染净化和太阳能能源转化方面都展现 出了良好的应用前景 ${ }^{[11 ~ 13]}$. 开发可见光催化剂的方 法可分为 2 大类: (1) 是对紫外光响应型的宽带隙光 催化材料进行改性, 如有非金属掺杂 ${ }^{[14]}$ 、半导体复 合 ${ }^{[15]}$ 、引人缺陷 ${ }^{[16]}$ 等方法; (2) 是设计和开发窄带隙
的可见光响应光催化剂 $[12,17,18]$.

近年来, $\mathrm{g}-\mathrm{C}_{3} \mathrm{~N}_{4}$ 作为一种新型可见光响应的非金 属半导体光催化材料引起了研究者的广泛关注, 因 其独特的电子能带结构和优异的稳定性已被广泛应 用于能源领域和环境光催化领域 ${ }^{[19 ~ 23]}$. 在现有报道 中, 一般针对粉体 $\mathrm{g}-\mathrm{C}_{3} \mathrm{~N}_{4}$ 进行制备、结构表征和性能 测试. 当粉体 $g-\mathrm{C}_{3} \mathrm{~N}_{4}$ 应用于实际的空气净化中时会 受到限制. 首先, 在气相反应过程中粉体光催化剂容 易受到气流冲刷而流失; 其次, 流失的光催化剂在气 流中会造成二次污染 ${ }^{[24]}$. 因此, 需要发展基于 $\mathrm{g}-\mathrm{C}_{3} \mathrm{~N}_{4}$ 的负载技术, 实现光催化剂在载体表面的牢固负载, 解决粉体光催化剂流失的问题，这是从实验室走向 应用的关键性技术问题 ${ }^{[25,26]}$.

本文以多孔 $\mathrm{Al}_{2} \mathrm{O}_{3}$ 泡沫陶瓷为载体, 开发了一种 溶解-再结晶-热处理原位负载工艺, 实现了 $\mathrm{g}-\mathrm{C}_{3} \mathrm{~N}_{4}$ 的 
牢固负载. 系统研究了在不同热处理时间下负载型 $\mathrm{g}-\mathrm{C}_{3} \mathrm{~N}_{4}$ 的微结构特征和光催化性能. $\mathrm{Al}_{2} \mathrm{O}_{3}$ 泡沫陶瓷 是一种比传统平板载体优越的优质光催化剂载体, 它的引人不仅增强了气体的紊流, 且提高了光利用 率 ${ }^{227,28]}$. 在可见光照射下, 负载型 $\mathrm{g}_{-} \mathrm{C}_{3} \mathrm{~N}_{4}$ 对低浓度 $\mathrm{NO}$ 去除率可达 $57.9 \%$, 为 $\mathrm{g}-\mathrm{C}_{3} \mathrm{~N}_{4}$ 在空气净化中的实 际应用奠定了技术支持和理论依据.

\section{1 实验}

(i) 催化剂的负载工艺. 称量 $40 \mathrm{~g}$ 二氰二胺于氧 化铝坩埚 $(160 \mathrm{~mm} \times 160 \mathrm{~mm} \times 78 \mathrm{~mm})$ 中, 加人 $600 \mathrm{~mL}$ 去 离子水, 搅拌 $15 \mathrm{~min}$, 使二氧二胺全部溶解. 将 $\mathrm{Al}_{2} \mathrm{O}_{3}$ 泡沫陶瓷(150 mm× $150 \mathrm{~mm} \times 22 \mathrm{~mm}, 200 \mathrm{~g}$, 苏州德 金陶瓷新材料有限公司, 中国)放人马弗炉中, 于 $550^{\circ} \mathrm{C}$ 下预处理 $30 \mathrm{~min}$. 再将预处理之后的 $\mathrm{Al}_{2} \mathrm{O}_{3}$ 泡沫 陶瓷浸没于二氰二胺溶液中, 随后将坩埚置于 $80^{\circ} \mathrm{C}$ 烘箱中, 进行二氧二胺的重结晶过程. 待水分全部烘 干之后, 白色的二氧二胺在多孔泡沫陶瓷内外表面 重新结晶. 最后将坩埚置于马弗炉中, 以 $15^{\circ} \mathrm{C} / \mathrm{min}$ 的 速率升温至 $550^{\circ} \mathrm{C}$ 煅烧制备样品, 热处理时间分别设 定为 $0.5,1,2,4$ 和 $6 \mathrm{~h}$, 以研究不同热处理时间对负载 型 $\mathrm{g}-\mathrm{C}_{3} \mathrm{~N}_{4}$ 光催化活性的影响, 制备得到的样品分别 标记为 CN-0.5 h, CN-1 h, CN-2 h, CN-4 h 和CN-6 h. $\mathrm{Al}_{2} \mathrm{O}_{3}$ 泡沫陶瓷如图 1(a) 所示. 在不同温度下负载
$\mathrm{g}-\mathrm{C}_{3} \mathrm{~N}_{4}$ 之后所得负载型催化剂的照片，如图 1(b) (f) 所示.

(ii) 催化剂的表征. 采用X射线衍射仪(XRD, Model D/max RA, 日本)对样品进行物相分析. 样品 的形态和结构通过扫描电子显微镜 (SEM, JEOL model JSM-6490, 日本) 和透射电子显微镜 (TEM, JEM-2010, 日本) 进行测试. 采用 $\mathrm{N}_{2}$ 吸附 - 脱附仪 (ASAP 2020, Micromeritics, 美国)测定样品的比表面 积和孔结构. 样品的光学性质用带有积分球的紫外 可见漫反射仪(UV-vis DRS, UV-2450, Shimadzu, 日 本)和苂光光谱(PL, F-7000, 日本)来分析. 采用热重差示扫描量热法 (TG-DSC, NETZSCH STA 409 $\mathrm{PC} / \mathrm{PG}$, 德国)测定前驱体在热处理过程中质量和热 量的变化, $20 \mathrm{mg}$ 二氧二胺至于氧化铝坩埚中, 升温 速率为 $15^{\circ} \mathrm{C} / \mathrm{min}$.

(iii) 催化剂的活性评估. 采用连续流动反应器 评估负载型 $\mathrm{g}-\mathrm{C}_{3} \mathrm{~N}_{4}$ 可见光催化去除 $\mathrm{ppb}$ 水平 $\mathrm{NO}$ 来评 价 $\mathrm{g}-\mathrm{C}_{3} \mathrm{~N}_{4}$ 的性能. 反应器由不锈钢和覆盖在其上方 的石英玻璃制成, 容积为 $4.5 \mathrm{~L}(30 \mathrm{~cm} \times 15 \mathrm{~cm} \times 10 \mathrm{~cm})$. 在反应器上方垂直放置一个 $150 \mathrm{~W}$ 的金卤灯, 采用截 止滤光片 (大于 $420 \mathrm{~nm}$ )去除紫外光. 将负载有 $\mathrm{g}-\mathrm{C}_{3} \mathrm{~N}_{4}$ 的 $\mathrm{Al}_{2} \mathrm{O}_{3}$ 泡沫陶瓷置于反应器中进行活性测试. $\mathrm{NO}$ 气 体来自浓度为 $100 \mathrm{mg} / \mathrm{m}^{3}$ 的 $\mathrm{NO}$ 标准气. 通过与空气混 合稀释到 $600 \mu \mathrm{g} / \mathrm{m}^{3}$. 气流经过气体搅拌器预混合
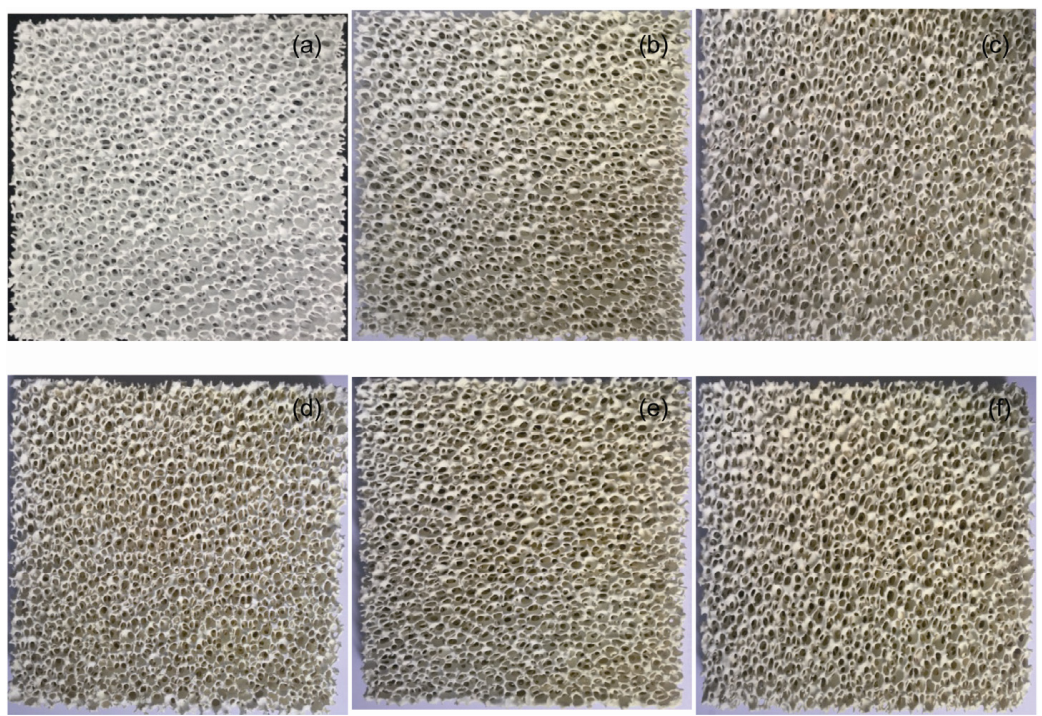

图 1 (网络版彩色) $\mathrm{Al}_{2} \mathrm{O}_{3}$ 泡沫陶瓷和不同热处理时间下负载型 $\mathrm{g}_{-} \mathrm{C}_{3} \mathrm{~N}_{4}$ 的照片. (a) $\mathrm{Al}_{2} \mathrm{O}_{3}$ 陶瓷; (b) CN-0.5 h; (c) CN-1 h; (d) CN-2 h; (e) CN-4 h; (f) CN-6 h

Figure 1 (Color online) The photo of $\mathrm{Al}_{2} \mathrm{O}_{3}$ ceramic (a) and the immobilized g-C $\mathrm{N}_{4}$ samples, CN-0.5 h (b), CN-1 h (c), CN-2 h (d), CN-4 h (e) and CN-6 $\mathrm{h}(\mathrm{f})$ 
后, 通人反应器中, 气体总流速为 $2.4 \mathrm{~L} / \mathrm{min}$, 相对湿 度为 $60 \%$. 待 $\mathrm{NO}$ 气体在样品表面达到吸附-脱附平衡 后再开灯. 通过化学发光氮氧化物分析仪 (Thermo Scientific, 42i-TL, 美国)连续监测 $\mathrm{NO}, \mathrm{NO}_{2}$ 和 $\mathrm{NO}_{x}\left(\mathrm{NO}_{x}\right.$ 指的是 $\mathrm{NO}$ 和 $\mathrm{NO}_{2}$ 的混合气)的浓度. 氮氧化物的去除 率 $(\eta)$ 可用下式计算:

$$
\eta(\%)=\left(1-C / C_{0}\right) \times 100 \%,
$$

其中, $C$ 表示开灯后反应器出口处的 $\mathrm{NO}$ 浓度, $C_{0}$ 表示 达到吸附-脱附平衡时的NO浓度.

\section{2 结果与讨论}

\section{1 表面和微观结构分析}

图2为不同热处理时间下负载样品的XRD图谱. 从图中可以看出, 各样品的衍射峰相似. 在 $27.3^{\circ}$ 处 的峰值最强, 对应(002)晶面, 该衍射峰是属于芳香 物的层间堆积, 产物具有类石墨的层状结构. 另一个 较弱的特征峰出现在 $13.1^{\circ}$ 左右, 对应(100)晶面, 该 衍射峰属于melon类物质的特征峰, 对应3-s-三嗪结 构 ${ }^{[29,30]}$. 进一步观察(002)主峰的强度, 可以发现, CN-6 h的主峰比CN-0.5 h 的主峰要强, 这表明热处理 时间较长的光催化剂, 其结晶度较高.

$\mathrm{Al}_{2} \mathrm{O}_{3}$ 陶瓷及不同负载型样品的SEM图像, 如图 3所示. 图3(a)和(b)显示了 $\mathrm{Al}_{2} \mathrm{O}_{3}$ 泡沫陶瓷的表面结 构，可以看出晶体粒子间相互堆积形成了随机分布 的孔状结构, 表面较为光滑. 图3(c) (1)是不同热处 理时间下负载型 $\mathrm{g}-\mathrm{C}_{3} \mathrm{~N}_{4}$ 样品的 SEM图. 从图中可以

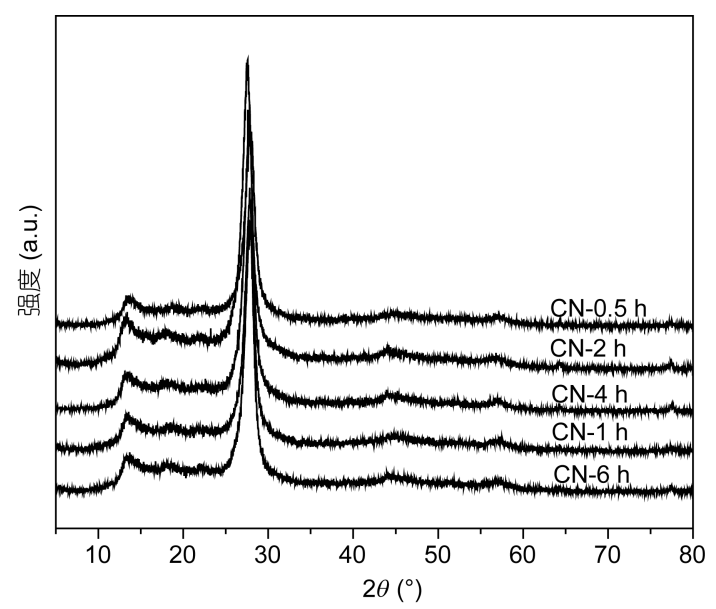

图 2 不同热处理时间下负载型 $\mathrm{g}-\mathrm{C}_{3} \mathrm{~N}_{4}$ 的 XRD 图谱

Figure 2 XRD pattern of $g-\mathrm{C}_{3} \mathrm{~N}_{4}$ loaded on the $\mathrm{Al}_{2} \mathrm{O}_{3}$ ceramic foam treated by different time

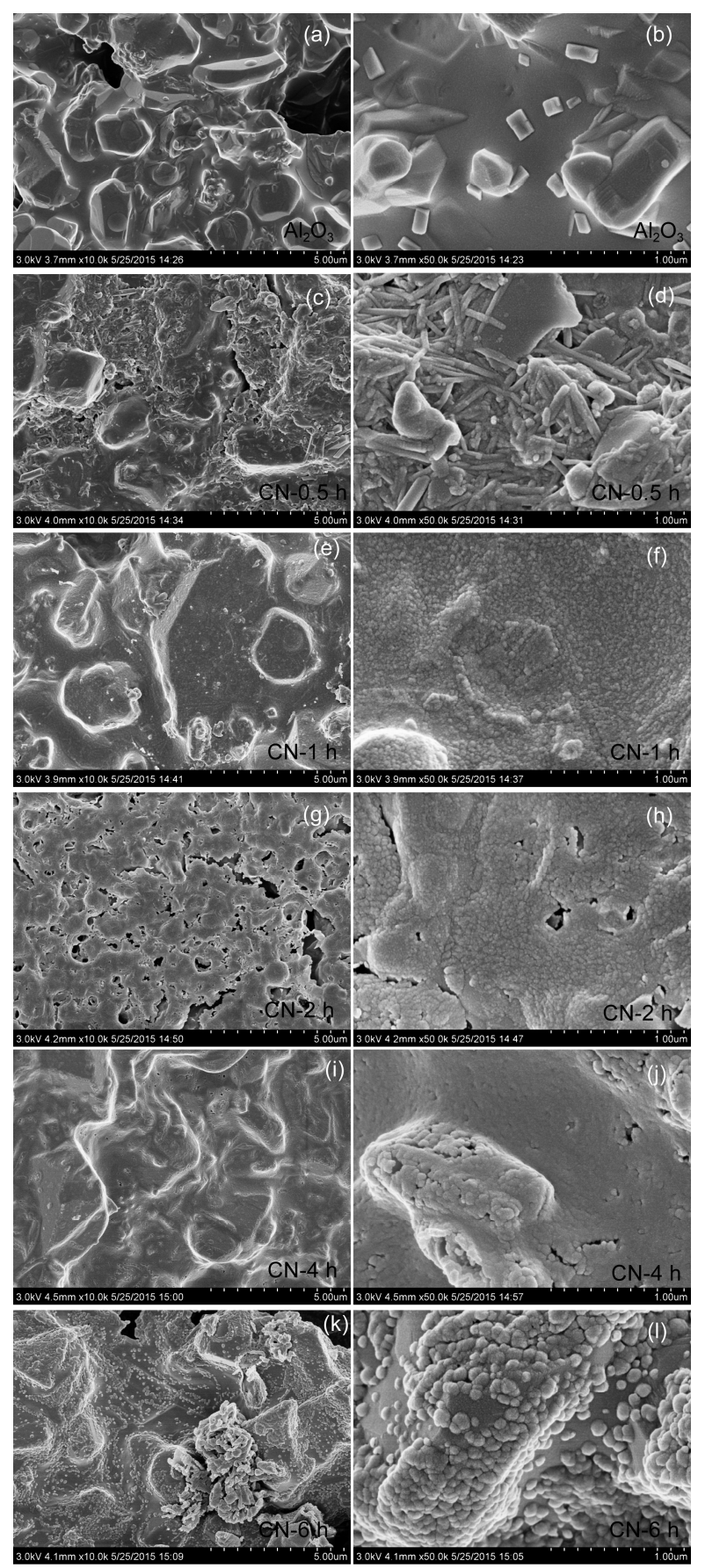

图 3 负载型 $\mathrm{g}-\mathrm{C}_{3} \mathrm{~N}_{4}$ 的 $\mathrm{SEM}$ 图像. (a, b) $\mathrm{Al}_{2} \mathrm{O}_{3}$ 陶瓷; (c, d) CN-0.5 h; (e, f) $\mathrm{CN}-1 \mathrm{~h}$; (g, h) CN-2 h; (i, j) CN-4 h; (k, l) CN-6 h

Figure 3 SEM images of the immobilized $\mathrm{g}_{-} \mathrm{C}_{3} \mathrm{~N}_{4}$ samples, $\mathrm{Al}_{2} \mathrm{O}_{3}$ ceramic (a, b), CN-0.5 h (c, d), CN-1 h (e, f), CN-2 h (g, h), CN-4 h (i, j) and $\mathrm{CN}-6 \mathrm{~h}(\mathrm{k}, 1)$

观察到, $\mathrm{Al}_{2} \mathrm{O}_{3}$ 泡沫陶瓷表面被二氰二胺热解形成的 g- $\mathrm{C}_{3} \mathrm{~N}_{4}$ 产物覆盖. 图3(c)和(d)显示了热处理 $0.5 \mathrm{~h}$ 时得 到样品的表面结构. 可以观察到, 二氰二胺在 $\mathrm{Al}_{2} \mathrm{O}_{3}$ 泡沫陶瓷表面聚合不完全，产物在表面的负载不均 
匀. 当热处理时间延长至 $1 \mathrm{~h}$, 如图 3(e) 和(f) 所示, $\mathrm{g}-\mathrm{C}_{3} \mathrm{~N}_{4}$ 在载体上分布均匀, 形成表面较为连续光滑 的层状结构. 进一步延长时间至 2 和 $4 \mathrm{~h}$, 可以观察到 载体表面的 $\mathrm{g}-\mathrm{C}_{3} \mathrm{~N}_{4}$ 变得更加均匀, 并形成了孔状结 构 (图 3(g) (j)). 孔结构的形成可归因于热处理过程 中 $\mathrm{g}-\mathrm{C}_{3} \mathrm{~N}_{4}$ 分解造成的, 因为随着热解时间的延长, $\mathrm{g}-\mathrm{C}_{3} \mathrm{~N}_{4}$ 会形成连续光滑且均匀的层状结构, 由于层 状结构间的堆积作用便会有孔结构的形成; 另一方 面是因为在热解过程中层状结构会逐渐剥落分离, 剥落分离的过程并不是在表面整体进行, 而是分为 每一个小区域进行, 从而形成了孔结构. 但当热处理 时间增加到 $6 \mathrm{~h}$ 时, 剥落分离的一个个小区域的孔状 结构会逐渐消失, 形成了如图 3(k) 和(l)的结构, 这是 由于过长的热处理时间使得 $\mathrm{g}^{-} \mathrm{C}_{3} \mathrm{~N}_{4}$ 进一步分解, 形 成不连续的颗粒状 $\mathrm{g}-\mathrm{C}_{3} \mathrm{~N}_{4} . \mathrm{g}-\mathrm{C}_{3} \mathrm{~N}_{4}$ 在载体表面的负载 因此变得不均匀, 部分载体暴露出来. 这些结果表明 在适当的热处理时间下, 层状结构的 $\mathrm{g}-\mathrm{C}_{3} \mathrm{~N}_{4}$ 可均匀 地负载在载体上. SEM图像分析表明, $\mathrm{g}-\mathrm{C}_{3} \mathrm{~N}_{4}$ 可通过 原位负载的方法成功地负载在 $\mathrm{Al}_{2} \mathrm{O}_{3}$ 泡沫陶瓷上.

采用TG-DSC模拟二㲵二胺的热处理过程(图4), 热处理范围为常温至 $550^{\circ} \mathrm{C}$, 升温速率为 $15^{\circ} \mathrm{C} / \mathrm{min}$. $\mathrm{DSC}$ 曲线中位于 $219^{\circ} \mathrm{C}$ 的峰对应二氧二胺的熔点. 在 $230 \sim 340^{\circ} \mathrm{C}$ 出现了位于 $267^{\circ} \mathrm{C}$ 的放热峰, 该温度对应 二氭二胺反应生成三聚氭胺的转化温度, 该温度段 的质量损失为 $26.7 \% .363^{\circ} \mathrm{C}$ 的放热峰对应三聚氧胺 的升华温度，在 $340 \sim 384^{\circ} \mathrm{C}$, 质量损失为 $10.1 \%$. $420^{\circ} \mathrm{C}$ 的放热峰对应三聚氭胺反应生成蜜勒胺的转化 温度. $500^{\circ} \mathrm{C}$ 放热峰为蜜勒胺转化生成 $g-\mathrm{C}_{3} \mathrm{~N}_{4}$ 的温度.

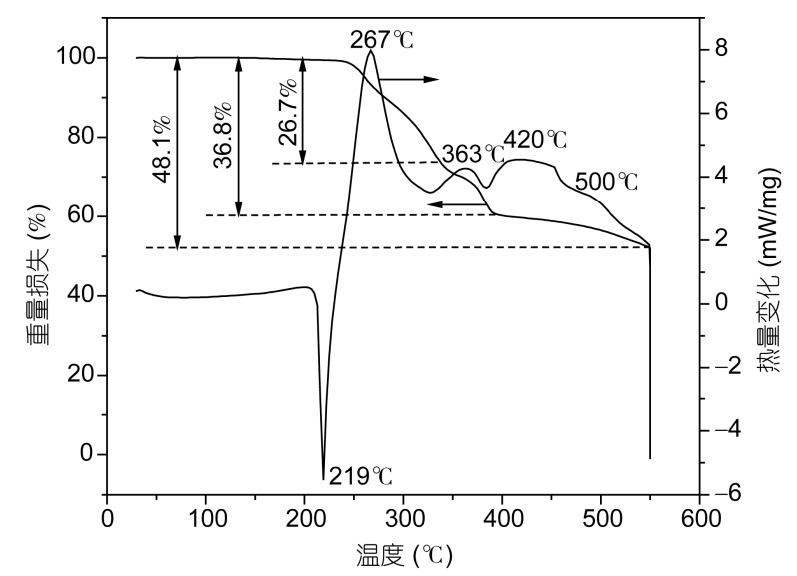

图 4 热处理二氰二胺的 TG-DSC 曲线

Figure 4 TG-DSC thermograms for heating dicyandiamide.
在 $384 \sim 550^{\circ} \mathrm{C}$, 质量损失为 $11.3 \%$. TG-DSC结果动态 显示了热处理二氧二胺生成 $\mathrm{g}-\mathrm{C}_{3} \mathrm{~N}_{4}$ 的过程. $550{ }^{\circ} \mathrm{C}$ 下 延长热处理时间, 生成的 $\mathrm{g}-\mathrm{C}_{3} \mathrm{~N}_{4}$ 全部分解.

通过称取载体在负载前后的质量差, 得到 $\mathrm{g}-\mathrm{C}_{3} \mathrm{~N}_{4}$ 催化剂的负载量. 如图 5所示, 在载体质量保 持不变基础上下, $\mathrm{g}-\mathrm{C}_{3} \mathrm{~N}_{4}$ 催化剂在泡沫陶瓷表面的负载 量随着热处理时间的延长, 催化剂的负载量从 $20.6 \mathrm{~g}$ 减 小至 $10.4 \mathrm{~g}$. 这是由于热处理时间延长, $\mathrm{g}-\mathrm{C}_{3} \mathrm{~N}_{4}$ 在载 体表面逐步分解引起的, 与SEM的观察结果一致.

为进一步研究 $\mathrm{g}-\mathrm{C}_{3} \mathrm{~N}_{4}$ 在载体表面的分布情况, 对 CN-4 h样品进行了选区元素成像能谱测试. 如图6 所示, 在选定的区域内, 可以检测到 $\mathrm{Al}, \mathrm{Si}, \mathrm{O}, \mathrm{C}$ 和 $\mathrm{N}$ 元素. 其中 $\mathrm{Al}, \mathrm{Si}$ 和 $\mathrm{O}$ 元素来自于载体本身, $\mathrm{C}$ 和 $\mathrm{N}$ 元 素来自负载的 $\mathrm{g}-\mathrm{C}_{3} \mathrm{~N}_{4}$. 从元素分布来看, $\mathrm{g}-\mathrm{C}_{3} \mathrm{~N}_{4}$ 均匀 地分布在 $\mathrm{Al}_{2} \mathrm{O}_{3}$ 陶瓷表面, 这与 $\mathrm{SEM}$ 观察结果一致.

将 $\mathrm{g}-\mathrm{C}_{3} \mathrm{~N}_{4}$ 从载体表面剥落下来用于 TEM分析. 如图7所示, 当热处理时间为 $1 \mathrm{~h}$ 时, 样品为块状, 层 厚度大(图7(a)和(b)). 随着热解时间的延长, g- $\mathrm{C}_{3} \mathrm{~N}_{4}$ 层状结构逐渐剥落、分离, 层状结构变得更薄, 且变 得多孔疏松(图7(c) (f)), 这是由于 $\mathrm{g}-\mathrm{C}_{3} \mathrm{~N}_{4}$ 的层状结构 间是通过 $\mathrm{CN}$ 环间的氢键连接，由于氢键间的键能很 弱, 在热氧化作用下易发生断裂，相应的 CN环也会 随之断裂, 从而导致 $\mathrm{g}-\mathrm{C}_{3} \mathrm{~N}_{4}$ 的层状结构逐渐剥落、分 离为更薄的结构, 并且随着热解过程的进行, $g-\mathrm{C}_{3} \mathrm{~N}_{4}$ 较大的层状结构也会发生分裂作用以降低表面能, 因此也使得 $\mathrm{g}-\mathrm{C}_{3} \mathrm{~N}_{4}$ 的结构发生变化 ${ }^{[31,32]}$. 当热处理 时间增加到 $6 \mathrm{~h}$ 时, 层状结构尺寸进一步减小, 呈颗 粒堆积状态.

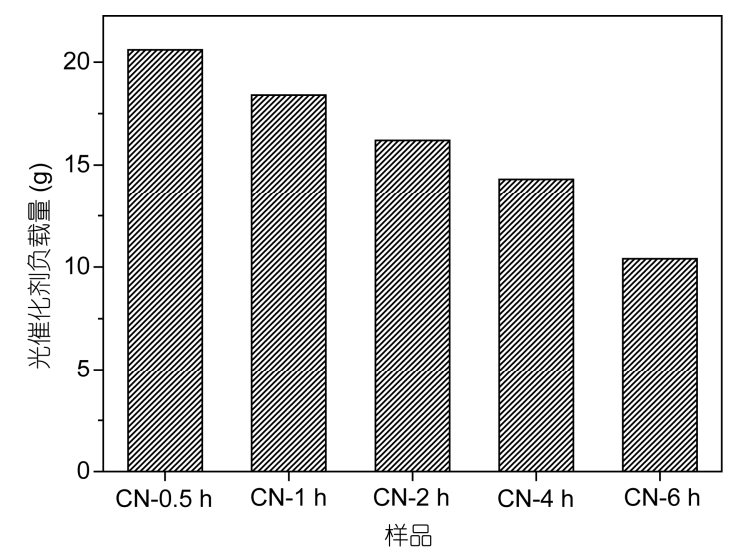

图 5 不同热处理时间下 $\mathrm{g}-\mathrm{C}_{3} \mathrm{~N}_{4}$ 催化剂的负载量示意图

Figure 5 The illustration of the $\mathrm{g}-\mathrm{C}_{3} \mathrm{~N}_{4}$ catalysts immobilized under different temperatures 


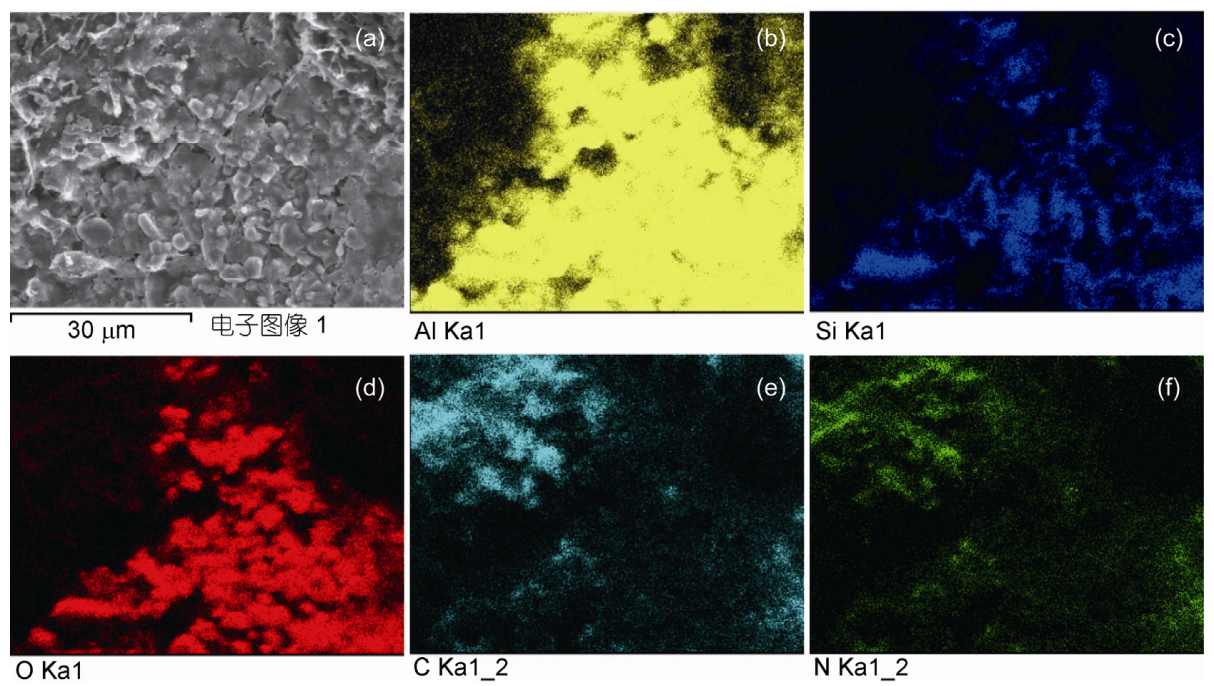

图 6 (网络版彩色)负载型 $\mathrm{g}-\mathrm{C}_{3} \mathrm{~N}_{4}(\mathrm{CN}-4 \mathrm{~h}$ ) 的元素成像能谱, 成像区域(a), $\mathrm{Al}$ (b), $\mathrm{Si}(\mathrm{c}), \mathrm{O}(\mathrm{d}), \mathrm{C}(\mathrm{e})$ 和 $\mathrm{N}(\mathrm{f})$

Figure 6 (Color online) The EDX elemental mapping of immpobilized $\mathrm{g}-\mathrm{C}_{3} \mathrm{~N}_{4}$, mapping area (a), $\mathrm{Al}(\mathrm{b}), \mathrm{Si}(\mathrm{c}), \mathrm{O}$ (d), $\mathrm{C}$ (e) and $\mathrm{N}$ (f)
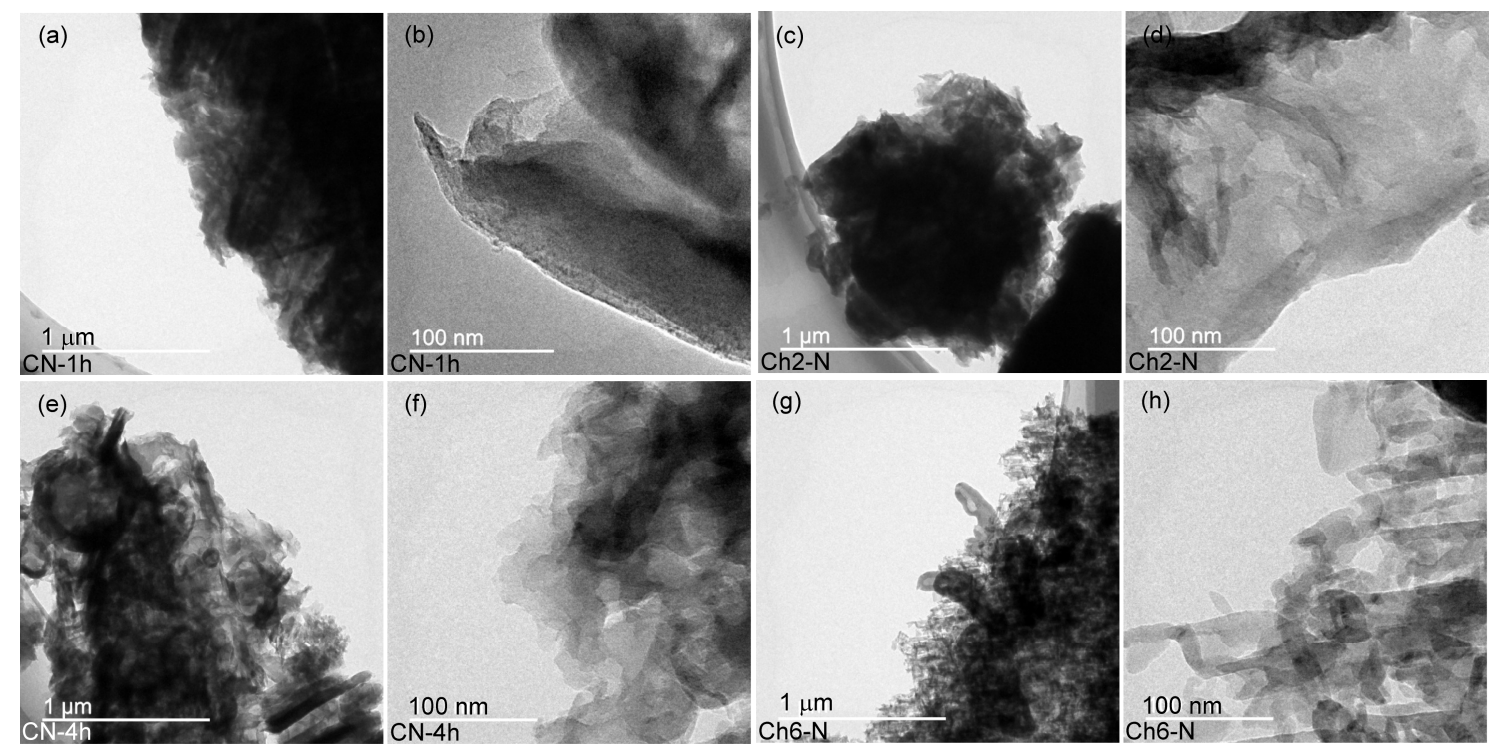

图 7 g-C $\mathrm{C}_{3} \mathrm{~N}_{4}$ 样品的 TEM 图像. (a, b) CN-1 h; (c, d) CN-2 h; (e, f) CN-4 h; (g, h) CN-6 h

Figure 7 TEM images of $g-\mathrm{C}_{3} \mathrm{~N}_{4}$ samples, $\mathrm{CN}-1 \mathrm{~h}(\mathrm{a}, \mathrm{b}), \mathrm{CN}-2 \mathrm{~h}(\mathrm{c}, \mathrm{d}), \mathrm{CN}-4 \mathrm{~h}(\mathrm{e}, \mathrm{f})$ and $\mathrm{CN}-6 \mathrm{~h}(\mathrm{~g}, \mathrm{~h})$

图8 是不同 $\mathrm{g}-\mathrm{C}_{3} \mathrm{~N}_{4}$ 样品的 $\mathrm{N}_{2}$ 吸附-脱附等温曲线及 Barrett-Joyner-Halenda(BJH)孔径分布曲线. 从图 8(a) 中可知, 样品的 $\mathrm{N}_{2}$ 吸附-脱附等温线都属于 IV 型等温 线(按BDDT分类), 这是介孔(2 50 nm)材料最普遍的 类型, 滞回环属于H3型(按IUPAC分类) ${ }^{[33]}$, 表明样品 中存在由片状物聚集而形成的孔结构. 从图 8(b)以及 TEM图中都可看出, 其中样品 CN-4 h的滞回环向相对 压力更低的区域移动最多, 说明形成了孔径较大的介 孔. 表1给出了不同样品的比表面积、孔径大小以及
NO的去除率. 随着热处理时间的增加(从 $0.5 \sim 4 \mathrm{~h}$ )，样 品的表面积、孔径大小相应增加，这SEM和TEM观测到 的结果一致. 随着热处理时间增加至 $6 \mathrm{~h}, \mathrm{~g}-\mathrm{C}_{3} \mathrm{~N}_{4}$ 开始 分解，颗粒之间相互聚集，导致比表面积下降.

\section{2 光物理性质}

图9(a)是不同热处理时间下 $g-\mathrm{C}_{3} \mathrm{~N}_{4}$ 样品的紫外可 见漫反射光谱. $g-\mathrm{C}_{3} \mathrm{~N}_{4}$ 的吸收边随着炦烧时间的不同 略有变化，吸收带边在 $462 \mathrm{~nm}$. 当热处理时间从 $1 \mathrm{~h}$ 

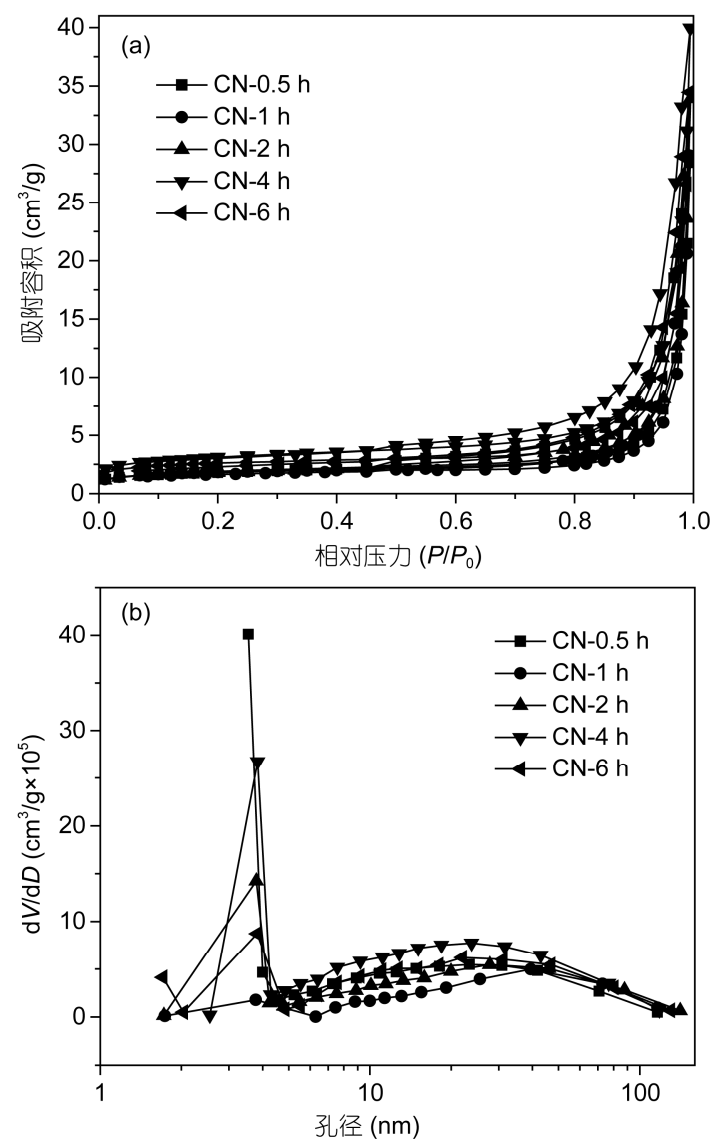

图 8 不同热处理时间下 $g-\mathrm{C}_{3} \mathrm{~N}_{4}$ 样品的 $\mathrm{N}_{2}$ 吸附-脱附曲线(a)和孔径 分布曲线(b)

Figure $8 \quad \mathrm{~N}_{2}$ adsorption-desorption isotherms (a) and corresponding pore-size distribution curves (b) of $\mathrm{g}^{-} \mathrm{C}_{3} \mathrm{~N}_{4}$ treated by different time

表 $1 \mathrm{~g}-\mathrm{C}_{3} \mathrm{~N}_{4}$ 样品的比表面积、孔径大小以及 NO 去除率

Table 1 The surface areas, pore parameters and NO removal ratio of the samples

\begin{tabular}{cccc}
\hline 样品 & 比表面积 $\left(\mathrm{m}^{2} / \mathrm{g}\right)$ & 孔径大小 $\left(\mathrm{cm}^{3} / \mathrm{g}\right)$ & $\mathrm{NO}$ 去除率 $(\%)$ \\
\hline $\mathrm{CN}-0.5 \mathrm{~h}$ & 6 & 0.044 & 37.7 \\
$\mathrm{CN}-1 \mathrm{~h}$ & 6 & 0.045 & 45.5 \\
$\mathrm{CN}-2 \mathrm{~h}$ & 6 & 0.053 & 49.2 \\
$\mathrm{CN}-4 \mathrm{~h}$ & 10 & 0.061 & 57.9 \\
$\mathrm{CN}-6 \mathrm{~h}$ & 8 & 0.053 & 46.5 \\
\hline
\end{tabular}

增加到 $6 \mathrm{~h}$ 时, $\mathrm{g}-\mathrm{C}_{3} \mathrm{~N}_{4}$ 的吸收边发生红移后再蓝移, 这 与 $\mathrm{g}-\mathrm{C}_{3} \mathrm{~N}_{4}$ 样品结构变化密切相关(图3和图7).

图9(b)为不同热处理时间制备所得样品的室温 PL光谱图. 发光带约在 $460 \mathrm{~nm}$ 处, 与吸收带边一致. 随着热处理时间的增加, 光致发光光谱的强度逐渐 减弱. 这这表明, 热处理时间延长有利于光生电荷分
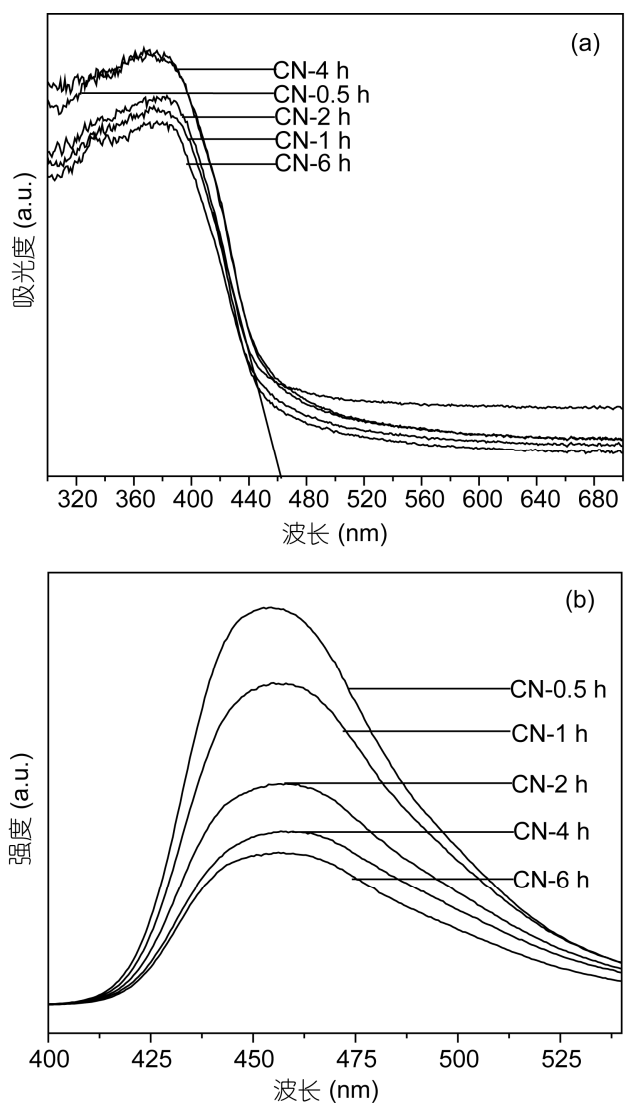

图 9 不同热处理时间下 $\mathrm{g}-\mathrm{C}_{3} \mathrm{~N}_{4}$ 样品的紫外可见漫反射光谱(a)和 PL 光谱(b)

Figure 9 UV-vis (a) and PL spectra (b) of g- $\mathrm{C}_{3} \mathrm{~N}_{4}$ samples treated by different time

离, 使得电子-空穴的复合率降低 ${ }^{[32]}$.

\section{3 可见光催化去除空气中NO的应用}

低浓度NO是室内和室外空气中的主要污染物之 一，光催化技术在净化 NO方面展现了良好的前 景 ${ }^{[34,35]} \cdot g-\mathrm{C}_{3} \mathrm{~N}_{4}$ 合适的禁带宽度能直接被可见光激 发，可通过在可见光照射条件下对低浓度NO的去除 率来评价负载型 $\mathrm{g}-\mathrm{C}_{3} \mathrm{~N}_{4}$ 的光催化性能. 根据 $\mathrm{g}-\mathrm{C}_{3} \mathrm{~N}_{4}$ 的 能带结构，可见光照射下产生的电子和空穴，电子可 以和 $\mathrm{O}_{2}$ 反应, 生成超氧自由基 $\left(\cdot \mathrm{O}_{2}^{-}\right)$与 $\mathrm{NO}$ 发生反应, 使其被氧化为终产物 $\mathrm{NO}_{3}^{-}$, 空穴可以直接参与氧化 $\mathrm{NO}$ 的反应 ${ }^{[25]}$, 达到去除 $\mathrm{NO}$ 的目的. 从图 10 可知, 当 热处理时间从 $0.5 \mathrm{~h}$ 增加到 $4 \mathrm{~h}$ 时 $\mathrm{NO}$ 去除率从 $37.7 \%$ 增 加到 $57.9 \%$, 这与样品的比表面积和光致发光强度一 致. 但当热处理时间增加到 $6 \mathrm{~h}$ 时，NO去除率下降. 虽然CN-6 h 的 PL强度最低, 但是其比表面积较小, 有效负载量较低, 影响了光催化性能的提高. 综合表 


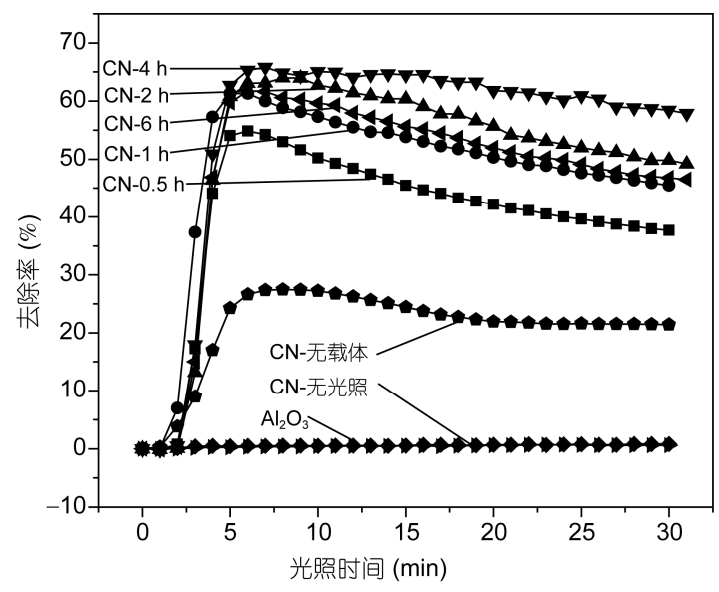

图 10 不同热处理时间下负载型 $\mathrm{g}-\mathrm{C}_{3} \mathrm{~N}_{4}$ 去除空气中 $\mathrm{NO}$ 的可见光催 化活性

Figure 10 Visible light photocatalytic activities of g- $\mathrm{C}_{3} \mathrm{~N}_{4}$ loaded on the $\mathrm{Al}_{2} \mathrm{O}_{3}$ ceramic foam treated by different time for removal of $\mathrm{NO}$ in air

征结果和性能测试结果, 热处理 $4 \mathrm{~h}$ 是制备负载型 $\mathrm{g}-\mathrm{C}_{3} \mathrm{~N}_{4}$ 最优时间. 样品 $\mathrm{CN}-4 \mathrm{~h}$ 的高效光催化活性可 归因于较高的比表面积及孔径(表1), 增强的电荷分 离效率(图9(b)). 较高的比表面积可以为光催化反应
提供更多的活性电位，增强的电荷分离效率有利于 产生更多的电子空穴对，有利于去除污染物.

本文报道的原位负载方法利用了 $\mathrm{g}-\mathrm{C}_{3} \mathrm{~N}_{4}$ 和 $\mathrm{Al}_{2} \mathrm{O}_{3}$ 之间的独特物理化学作用力, $\mathrm{g}-\mathrm{C}_{3} \mathrm{~N}_{4}$ 中 $\mathrm{N}$ 的孤对电子 占据 $\mathrm{Al}_{2} \mathrm{O}_{3}$ 中 $\mathrm{Al}$ 的空轨道, 增强了光催化剂和载体之 间的相互作用，实现了 $\mathrm{g}-\mathrm{C}_{3} \mathrm{~N}_{4}$ 在 $\mathrm{Al}_{2} \mathrm{O}_{3}$ 泡沫陶瓷上的 牢固负载 ${ }^{[25]}$, 解决了粉体光催化剂流失的问题. 这 是从实验室走向应用的关键环节, 为 $\mathrm{g}-\mathrm{C}_{3} \mathrm{~N}_{4}$ 在空气 净化中的应用奠定了理论和技术基础.

\section{3 结论}

利用光催化剂与载体之间的独特物理化学作用 力, 通过原位负载方法将 $\mathrm{g}-\mathrm{C}_{3} \mathrm{~N}_{4}$ 牢固地负载在 $\mathrm{Al}_{2} \mathrm{O}_{3}$ 泡沫陶瓷上. 合成的负载型 $\mathrm{g}-\mathrm{C}_{3} \mathrm{~N}_{4}$ 具有高效的 $\mathrm{NO}$ 去 除率，高达 $57.9 \%$ 。这归因于其较大的比表面积及 孔径, 较高的电荷分离效率. 本研究解决了 $\mathrm{g}-\mathrm{C}_{3} \mathrm{~N}_{4}$ 在空气净化应用的关键技术问题, 为利用 $\mathrm{g}-\mathrm{C}_{3} \mathrm{~N}_{4}$ 在空气净化中的实际应用提供了技术支持和理论 依据.

\section{参考文献}

1 Lammel G, Cape J N. Nitrous acid and nitrite in the atmosphere. Chem Soc Rev, 1996, 5: 361-369

2 Spicer C W. Photochemical atmospheric pollutants derived from nitrogen oxides. Atmos Environ, 1977, 11: 1089-1095

3 Zeng Z, Lu P, Li C, et al. Removal of NO by carbonaceous materials at room temperature: A review. Catal Sci Technol, 2012, 11: 2188-2199

$4 \mathrm{Chu} \mathrm{H,} \mathrm{Li} \mathrm{S} \mathrm{Y,} \mathrm{Chien} \mathrm{T} \mathrm{W.} \mathrm{The} \mathrm{absorption} \mathrm{kinetics} \mathrm{of} \mathrm{no} \mathrm{from} \mathrm{flue} \mathrm{gas} \mathrm{in} \mathrm{a} \mathrm{stirred} \mathrm{tank} \mathrm{reactor} \mathrm{with} \mathrm{KMnO}_{4} / \mathrm{NaOH}$ solutions. J Environ Sci Health Part A, 1998, 5: 801-827

5 Tang B, Li J, Wang Y L. Exploration of denitrification adsorbent made from fly ash (in Chinese). Environm Eng, 2006, 3: 45-46 [唐冰, 李坚，王艳磊，等. 应用粉煤灰制作脱氮吸附剂的探讨. 环境工程, 2006, 3: 45-46]

6 Lee I Y, Kim D W, Lee J B, et al. A practical scale evaluation of sulfated $\mathrm{V}_{2} \mathrm{O}_{5} / \mathrm{TiO}_{2}$ catalyst from metatitanic acid for selective catalytic reduction of $\mathrm{NO}$ by $\mathrm{NH}_{3}$. Chem Eng J, 2002: 267-272

7 Ye D Q. Treatment of nitrogen oxides in flue gas streams (in Chinese). Environm Prot Sci, 1999, 4: 1-4 [叶代启. 烟气中氮氧化物污染 的治理. 环境保护科学, 1999, 4: 1-4]

8 Sada E, Kumazawa $\mathrm{H}$, Kudo I, et al. Individual and simultaneous absorption of dilute $\mathrm{NO}$ and $\mathrm{SO}_{2}$ in aqueous slurries of $\mathrm{MgSO}_{3}$ with Fe(II) EDTA. Ind Eng Chem Process Design Develop, 1980: 377-382

9 Huang Y, Ho W K, Lee S C, et al. Effect of carbon doping on the mesoporous structure of nanocrystalline titanium dioxide and its solar-light-driven photocatalytic degradation of $\mathrm{NO}_{x}$. Langmuir, 2008, 7: 3510-3516

10 Ao C H, Lee S C, Yu J C. Photocatalyst $\mathrm{TiO}_{2}$ supported on glass fiber for indoor air purification: Effect of NO on the photodegradation of $\mathrm{CO}$ and $\mathrm{NO}_{2}$. J Photochem Photobiol A Chem, 2003, 1-3: 171-177

11 Zhang T, Zhang Y W, Zhang S Z, et al. Research and applications of visible light responsive narrow band gap semiconductor photocatalytic materials (in Chinese). Mater Rev, 2009, 3: 24-28 [张娅, 张悦炜, 张世著, 等. 可见光响应型窄带隙半导体光催化材料的研究 及应用进展. 材料导报, 2009, 3: 24-28]

12 Yan S C, Luo W J, Li Z S, et al. Progress in research of novel photocatalytic materials (in Chinese). Mater China, 2010, 1: 1-9 [i间世成, 罗文俊, 李朝升, 等. 新型光催化材料探索和研究进展. 中国材料进展, 2010, 1: 1-9]

13 Huang B B, Wang P, Zhang X Y, et al. Progress in research of visible light photocatalysis (in Chinese). Chin Sci Bull (Chin Ver), 2009, 54: 847 [黄柏标, 王朋, 张晓阳, 等. 可见光光催化研究新进展. 科学通报, 2009, 54: 847] 
14 Asahi R, Morikawa T, Ohwaki T, et al. Visible-light photocatalysis in nitrogen-doped titanium oxides. Science, 2001, 5528: 269-271

15 Issei $\mathrm{T}$, Hideki $\mathrm{K}$, Hisayoshi $\mathrm{K}$, et al. Photocatalytic $\mathrm{H}_{2}$ evolution reaction from aqueous solutions over band structure-controlled $(\mathrm{AgIn})_{x} \mathrm{Zn}_{2(1-x)} \mathrm{S}_{2}$ solid solution photocatalysts with visible-light response and their surface nanostructures. J Am Chem Soc, 2004, 41: 13406-13413

$16 \mathrm{Li} \mathrm{Q} \mathrm{Y,} \mathrm{Zhang} \mathrm{J} \mathrm{W,} \mathrm{Jin} \mathrm{Z} \mathrm{S,} \mathrm{et} \mathrm{al.} \mathrm{A} \mathrm{novel} \mathrm{TiO}_{2}$ with a large amount of bulk intrinsic defects-Visible-light-responded photocatalytic activity induced by foreign trap (in Chinese). Chin Sci Bull (Chin Ver), 2013, 58: 1007-1013 [李秋叶, 张纪伟, 金振声, 等. 含高浓度本 征缺陷的新型 $\mathrm{TiO}_{2}$ ：外来陷阱诱导产生可见光光催化活性. 科学通报, 2013, 58: 1007-1013]

$17 \mathrm{Ji} \mathrm{H} \mathrm{W}$, Ma W H, Huang Y P, et al. Progress in visible light responding $\mathrm{TiO}_{2}$ photocatalysts (in Chinese). Chin Sci Bull (Chin Ver), 2004 , 49: 2199-2204 [籍宏伟, 马万红, 黄应平, 等. 可见光诱导 $\mathrm{TiO}_{2}$ 光催化的研究进展. 科学通报, 2004, 49: 2199-2204]

18 Chen S Z, Zhang P Y, Zhu W P, et al. Progress in visible light responding photocatalysts (in Chinese). Prog Chem, 2004, 4: 613-619 [陈 崧哲, 张彭义, 祝万鹏, 等. 可见光响应光催化剂研究进展. 化学进展, 2004, 4: 613-619]

19 Cao S, Low J, Yu J, et al. Polymeric photocatalysts based on graphitic carbon nitride. Adv Mater, 2015, 13: 2150-2176

20 Dong F, Wu L, Sun Y, et al. Efficient synthesis of polymeric $\mathrm{g}-\mathrm{C}_{3} \mathrm{~N}_{4}$ layered materials as novel efficient visible light driven photocatalysts. J Mater Chem, 2011, 39: 15171-15174

21 Chu Z Y, Yuan B, Yan T N. Recent progress in photocatalysis of g- $\mathrm{C}_{3} \mathrm{~N}_{4}$ (in Chinese). J Inorg Mater, 2014, 8: 785-794 [楚增勇, 原博, 颜廷楠. g- $\mathrm{C}_{3} \mathrm{~N}_{4}$ 光催化性能的研究进展. 无机材料学报, 2014, 8: 785-794]

22 Zhang J S, Wang B, Wang X C. Chemical synthesis and applications of graphitic carbon nitride (in Chinese). Acta Phys Chim Sin, 2013, 1865-1876 [张金水, 王博, 王心晨. 石墨相氮化碳的化学合成及应用. 物理化学学报, 2013, 1865-1876]

23 Zhang J S, Wang B, Wang X C. Carbon nitride polymeric semiconduct for photocatalysis (in Chinese). Prog Chem, 2014, 26: 19-29 [张金 水, 王博, 王心晨. 氮化碳聚合物半导体光催化. 化学进展, 2014, 26: 19-29]

24 Yuan H, Sun Z, Liu H, et al. Immobilizing carbon nanotubes on sic foam as a monolith catalyst for oxidative dehydrogenation reactions. Chem Catal Chem, 2013, 7: 1713-1717

25 Dong F, Wang Z, Li Y, et al. Immobilization of polymeric $\mathrm{g}-\mathrm{C}_{3} \mathrm{~N}_{4}$ on structured ceramic foam for efficient visible light photocatalytic air purification with real indoor illumination. Environ Sci Technol, 2014, 17: 10345-10353

26 Kouamé A N, Masson R, Robert D, et al. $\beta$-SiC foams as a promising structured photocatalytic support for water and air detoxification. Catal Today, 2013, 209: 13-20

27 Yao Y, Ochiai T, Ishiguro H, et al. Antibacterial performance of a novel photocatalytic-coated cordierite foam for use in air cleaners. Appl Catal B Environ, 2011, 3-4: 592-599

28 Lin H, Valsaraj K T. Development of an optical fiber monolith reactor for photocatalytic wastewater treatment. J Appl Electrochem, 2005 , 7-8: 699-708

29 Dong F, Sun Y, Wu L, et al. Facile transformation of low cost thiourea into nitrogen-rich graphitic carbon nitride nanocatalyst with high visible light photocatalytic performance. Catal Sci Technol, 2012, 7: 1332-1335

30 Ge L. Synthesis and photocatalytic performance of novel metal-free g- $_{3} \mathrm{~N}_{4}$ photocatalysts. Mater Lett, 2011, 17: 2652-2654

31 Dong F, Wang Z, Sun Y, et al. Engineering the nanoarchitecture and texture of polymeric carbon nitride semiconductor for enhanced visible light photocatalytic activity. J Colloid Interface Sci, 2013, 401: 70-79

32 Dong F, Ou M Y, Jiang Y K, et al. Efficient and durable visible light photocatalytic performance of porous carbon nitride nanosheets for air purification. Ind Eng Chem Res, 2014, 53: 2318-2330

33 Sing K S. Reporting physisorption data for gas/solid systems with special reference to the determination of surface area and porosity (Recommendations 1984). Pure Appl Chem, 1985, 4: 603-619

34 Dong F, Li Q, Ho W K, et al. The mechanism of enhanced visible light photocatalysis with microstructurally optimized and graphene oxide coupled $(\mathrm{BiO})_{2} \mathrm{CO}_{3}$ (in Chinese). Chin Sci Bull, 2015, 60: 1915-1923 [董帆, 李秋燕, 何詠基, 等. 微结构优化与GO耦合大幅提 高 $(\mathrm{BiO})_{2} \mathrm{CO}_{3}$ 可见光催化性能的机制. 科学通报, 2015, 60: 1915-1923]

35 Dong F, Xiong T, Sun Y, et al. Controlling interfacial contact and exposed facets for enhancing photocatalysis via 2D-2D heterostructure. Chem Commun, 2015, 51: 8249-8252 


\title{
In situ immobilization of $\mathrm{g}-\mathrm{C}_{3} \mathrm{~N}_{4}$ on structured ceramic foam and visible light photocatalytic air purification
}

\author{
CUI Wen ${ }^{1}$, LI Hui ${ }^{1}$, SUN YanJuan ${ }^{1}$, DONG Fan ${ }^{1}$, HO Wing-Kei ${ }^{2} \&$ WU ZhongBiao ${ }^{3}$ \\ ${ }^{1}$ Chongqing Key Laboratory of Catalysis and Functional Organic Molecules, College of Environment and Resources, Chongqing Technology and \\ Business University, Chongqing 400067, China; \\ ${ }^{2}$ Department of Science and Environmental Studies, The Hong Kong Institute of Education, Hong Kong, China; \\ ${ }^{3}$ Department of Environmental Engineering, Key Laboratory of Polluted Environment Remediation and Ecological Health of Ministry of Education, \\ Zhejiang University, Hangzhou 310027, China
}

g- $\mathrm{C}_{3} \mathrm{~N}_{4}$ is a metal-free visible light photocatalyst with wide promising application. In this work, an in situ approach of dissolutionrecrystallization-thermal-treatment is developed for effective immobilization of $\mathrm{g}-\mathrm{C}_{3} \mathrm{~N}_{4}$ on the support using dicyandiamide as precursor. The immobilized g- $\mathrm{C}_{3} \mathrm{~N}_{4}$ on $\mathrm{Al}_{2} \mathrm{O}_{3}$ ceramic foam was firm enough owing to the special physicochemical interaction between $\mathrm{g}-\mathrm{C}_{3} \mathrm{~N}_{4}$ and $\mathrm{Al}_{2} \mathrm{O}_{3}$. The g- $\mathrm{C}_{3} \mathrm{~N}_{4}$ samples were analyzed by techniques. With a systematic investigation on the effects of different pyrolysis time on the microstructure and photocatalytic activity of $\mathrm{g}-\mathrm{C}_{3} \mathrm{~N}_{4}$, it was found that the immobilized $\mathrm{g}-\mathrm{C}_{3} \mathrm{~N}_{4}$ with a pyrolysis time of $4 \mathrm{~h}$ exhibited optimal NO removal ratio of $57.9 \%$. The outstanding activity can be ascribed to the increased surface area and porosity and promoted charge separation. This work could provide an in situ immobilization approach based on the interaction between the photocatalyst and support, as well as the technical support and theoretical basis in practical application of g- $\mathrm{C}_{3} \mathrm{~N}_{4}$ in air purification.

graphitic carbon nitride, in situ immobilization, visible light photocatalysis, charge separation, air purification

doi: 10.1360/N972015-00963 